\section{Baboon syndrome (SDRIFE) after valsartan/hydrochloroth- iazide intake for several years}

\section{Georgi Tchernev, Nikhil Oliveira, Lorraine Joseph Kandathil}

Onkoderma - Clinic for Dermatology, Venereology and Dermatologic Surgery, Sofia, Bulgaria

\begin{abstract}
Herein, we present a case of baboon syndrome/SDRIFE that occurred after intake of valsartan/hydrochlorothiazide for several years. The patient falls within the five diagnostic criteria and to the best of our knowledge, there have been no previous reports associating valsartan/hydrochlorothiazide with baboon syndrome/SDRIFE. Withdrawal of the anti-hypertensive drugs and initiation of topical and systemic steroids provided symptomatic relief and follow-up showed favourable results with complete remission of the cutaneous eruptions.
\end{abstract}

\section{Case Report}

A 57-year-old gentleman presented to our clinic with widespread erythematous rash around the trunk, flexural areas and buttocks. He was previously diagnosed with arterial hypertension in March 2015 and has since been put on a valsartan/hydrochlorothiazide 160/25 mg treatment regimen that has been ongoing for 6 years till date. He reported no other significant medical history, no other recent medications and reported no drug allergies. There were no other systemic signs or symptoms of concern. On examination, there were multiple, sharply demarcated, symmetrical eruptions that involved mostly the intertriginous areas (inguinal, buttocks and axillae) (Figure 1) as well as the flexural zones within the cubital/popliteal regions and the right arm (Figure 1b,d,e). The lesions were erythematous and appeared macerated as well as soft and oedematous on palpation. A blood work was immediately conducted as well as histology and microbiology to rule out concerns of necrotising fasciitis. At the time of investigation, both the eosinophilic count $(0.96$ G/L; Reference range: 0.03-0.044) and monocyte count $(0.93 \mathrm{G} / \mathrm{L}$; Reference

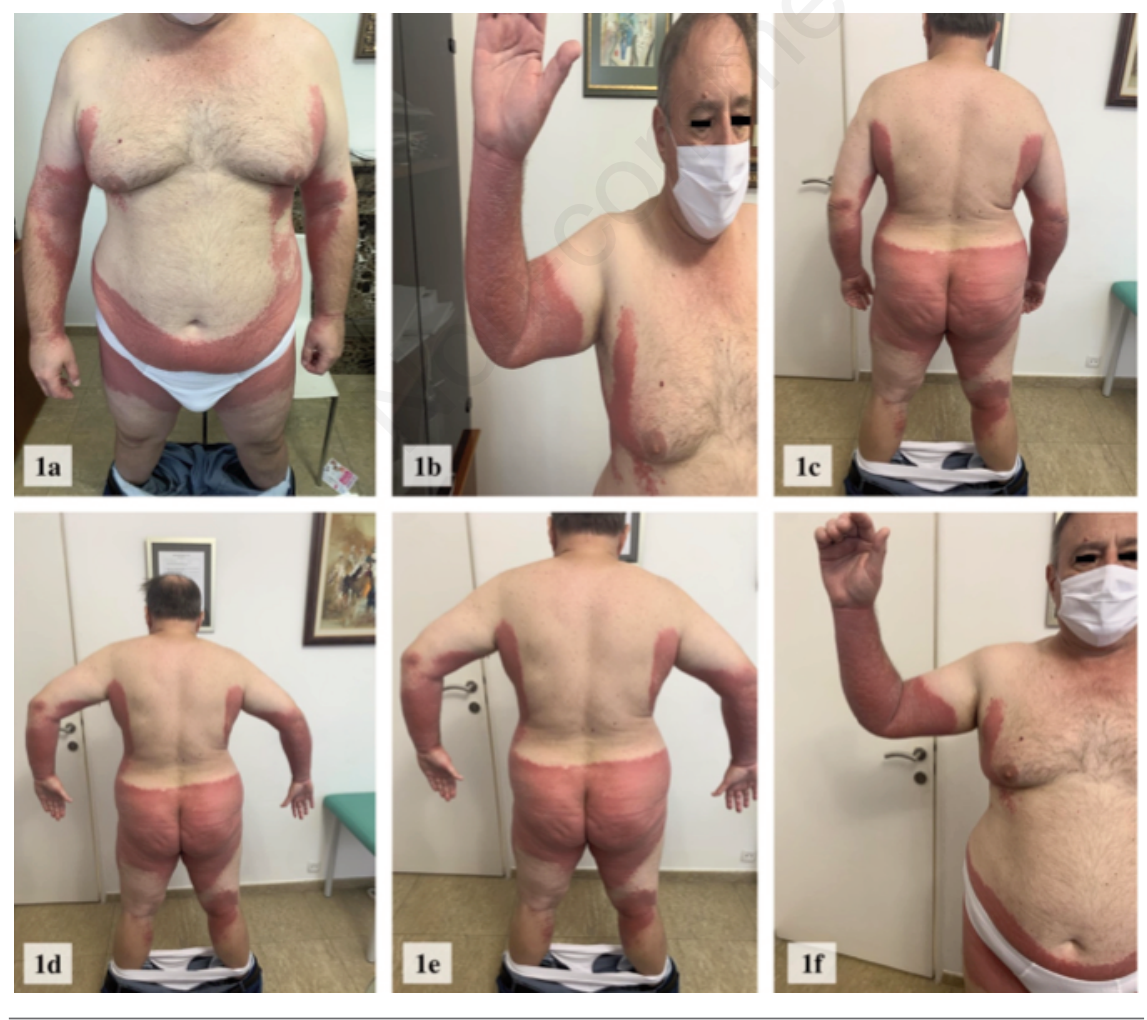

Figure 1. A-F) Images taken at the time of presentation demonstrating erythematous macerated rash in the intertriginous areas including buttocks, groin, upper thighs, flank and axillae.

Correspondence: Nikhil Oliveira, Onkoderma - Clinic for Dermatology, Venereology and Dermatologic Surgery, General Skobelev 26, 1606 Sofia, Bulgaria.

Tel.: +359876838005

E-mail: nikhil.oliveira@hotmail.com

Key words: Allergic contact dermatitis; Baboon syndrome; SDRIFE; Drug eruption; Valsartan.

Contributions: NO: conceptualization, methodology, investigation, writing - original and draft preparation. LJK: data curation, software, writing - original and draft preparation. GT: conceptualization, visualisation, supervision, writing- reviewing and editing.

Conflict of interest: The authors declare no potential conflict of interest.

Funding: None.

Ethical approval and consent to participate: All procedures performed were in accordance with the ethical standards of the institutional and/or national research committee(s) and with the Helsinki Declaration (as revised in 2013). Written informed consent was obtained for publication of report and accompanying images. A copy of the written consent is available on reasonable request.

Availability of data and material: Data and materials are available by the authors.

Please cite this article as: Tchernev $G$, Oliveira N, Kandathil LJ. Baboon syndrome (SDRIFE) after Valsartan/Hydrochloro-thiazide intake for several years. Dermatol Rep 2022;14:9412.

Received for publication: 27 October 2021. Accepted for publication: 29 November 2021.

This work is licensed under a Creative Commons Attribution-NonCommercial 4.0 International License (CC BY-NC 4.0).

(Copyright: the Author(s), 2022

Licensee PAGEPress, Italy

Dermatology Reports 2022; 14:9412

doi:10.4081/dr.2022.9412

Publisher's note: All claims expressed in this article are solely those of the authors and do not necessarily represent those of their affiliated organizations, or those of the publisher the editors and the reviewers. Any product that may be evaluated in this article or claim that may be made by its manufacturer is not guaranteed or endorsed by the publisher. 
range: 0.24-0.79) were elevated. All other blood parameters including blood cultures were negative. Gram stain also revealed no organisms (Figure 2). The subsequent biopsy reported mild interface dermatitis and minimal spongiosis, together with a mild superficial perivascular infiltrate consisting of neutrophils, monocytes and scattered eosinophils. The histological features alongside a clinical correlation were keeping in line with a diagnosis of Baboon syndrome (SDRIFE). The patient was consequently removed off his valsartan/hydrochlorothiazide regimen and promptly started on lercanidipine hydrochloride $10 \mathrm{mg}$ twice daily. Due to the macerated lesions, the patient was also immediately started on systemic broad spectrum antibiotics including clarithromycin $500 \mathrm{mg}$ (once daily for 10 days), dexamethasone $4 \mathrm{mg}$ twice daily for two weeks, followed by a reduction to $6 \mathrm{mg}$ daily for a week (4-0-2), after which it was tapered down to $2.5 \mathrm{mg}$ daily for the third week and finally to $2 \mathrm{mg}$ every second day for two weeks. He was also given esomeprazole $40 \mathrm{mg}$ twice daily to negate the side effects of dexamethasone. Local therapy was also administered including Advantan cream $0.1 \%$ 30g (methylprednisolone aceponate) and Fucicort (fusidic acid $20 \mathrm{mg}$ and betamethasone valerate $1 \mathrm{~g}$ ) to be applied twice daily for the macerated lesions. The patient's blood pressure was well controlled and monitored after a change in his therapy. He was discharged 2weeks after admission and remained in a good outcome at last follow-up with remarkable improvement of the original eruptions after discontinuation of valsartan/hydrochlorothiazide (Figure 3).

\section{Discussion and conclusions}

Baboon syndrome is an extremely rare condition characterised by well demarcated purpuric cutaneous eruptions within the gluteal and intertriginous areas that classically resembles that of red-bottomed baboons. ${ }^{1}$ Since its first description in the literature by Andersen et al in 1984 as a cutaneous reaction due to mercury and nickel exposure, there have been over 100 further reported cases to date with over $50 \%$ of cases being reported as drug-induced or drug related. ${ }^{2}$ Additionally, a new acronym of SDRIFE (Symmetrical drug-related intertriginous and flexural exanthema) was proposed to better define the condition, with a diagnostic criteria that included: a) Exposure to a systemically administered drug either at the first or repeated dose
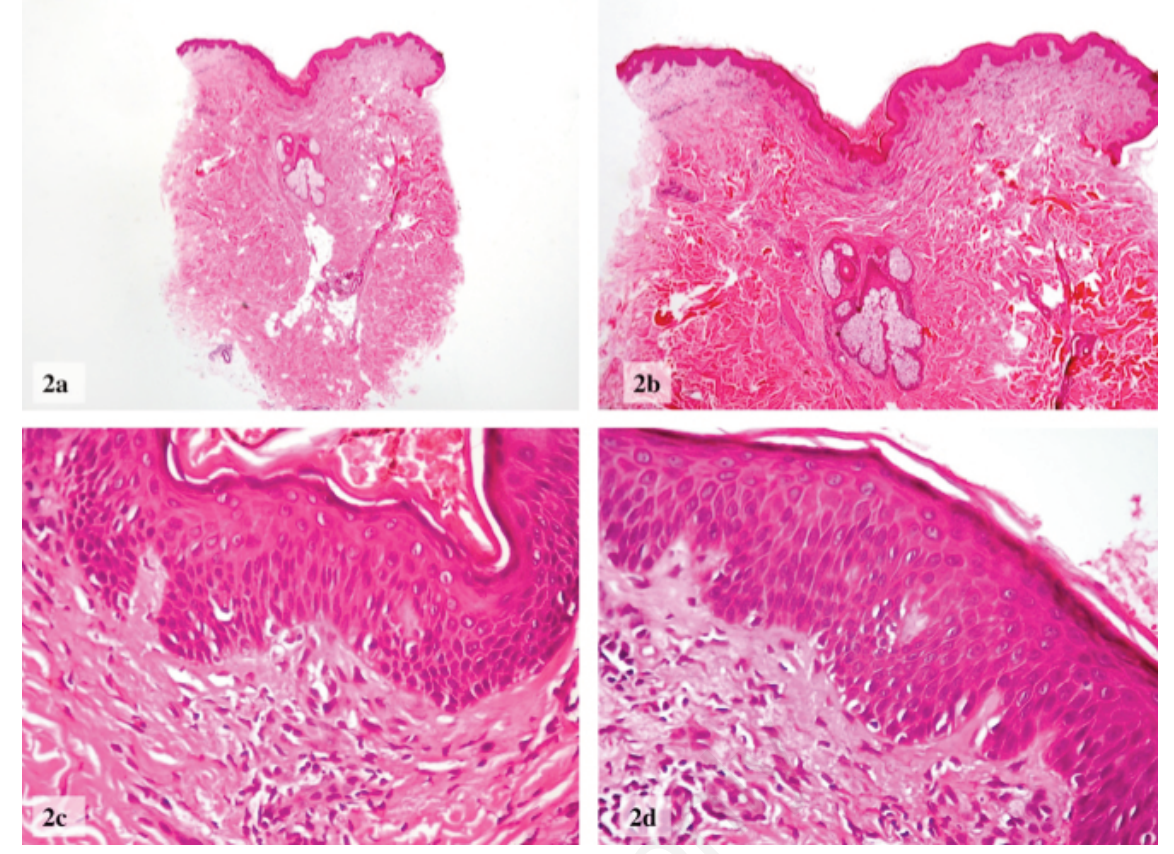

Figure 2. A-D) Photomicrographs of biopsy taken from the lesions that show mild interface dermatitis and minimal spongiosis together with a mild superficial perivascular infiltrate. The images do not warrant enough spongiosis for contact dermatitis.
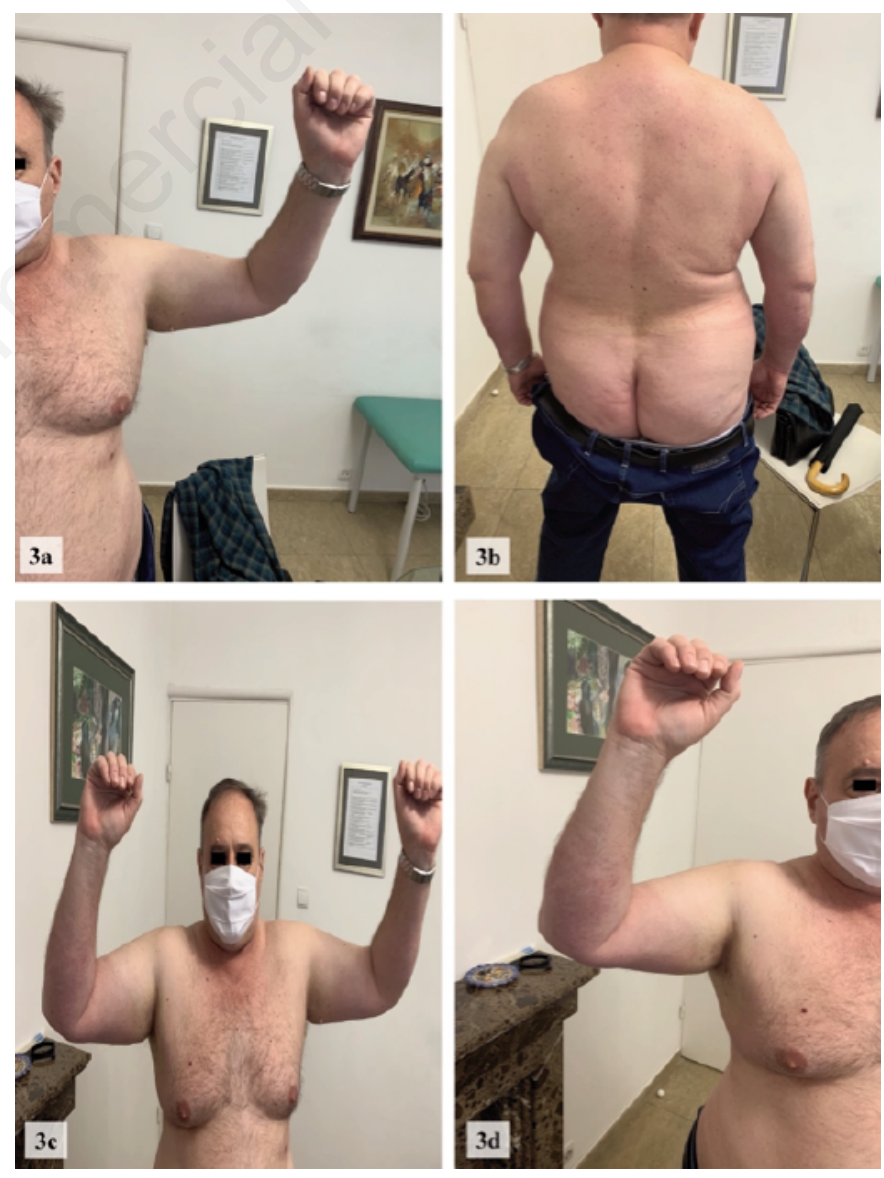

Figure 3. A-D Clinical images at 4-week follow up after discontinuation of valsartan/hydrochlorothiazide and administration of topical and systemic steroids and antibiotics. 
(excluding contact allergens); b) sharply demarcated erythema of the gluteal/perianal area and/or V-shaped erythema of the inguinal/perigenital area; c) involvement of at least another intertriginous/flexural localization; d) symmetry of affected areas; and e) absence of systemic symptoms and signs. $^{2}$

Of all the drug-induced agents reported, amoxillicin, ceftriaxone, penicillin and erythromycin have been identified as the most common causative agents of SDRIFE. ${ }^{3}$ Other non-antimicrobial agents including codeine, paracetamol, heparin, tamoxifen, antihistamines, iodine contrast media and chemotherapeutic agents including some monoclonal antibodies such as infliximab have also been indicted to cause this reactive condition. ${ }^{3,4}$ Many of such cases have shown to occur after several days of primary exposure to the drug without any prior sensitization..$^{3,4}$ However, contrary to this fact, cases have also been documented where drug-induced baboon syndrome/SDRIFE has occurred several years after initiation of use. ${ }^{3,4}$ Mofarrah and colleagues, for example, documented the first report of Baboon syndrome that occurred 8 years after tamoxifen use. ${ }^{3}$ It is extremely uncommon to see the possibility of such a reaction occurring after such a long period of time, albeit not impossible. This is of particular interest as the pathologic mechanisms are yet to be fully elucidated, with much onus being on the fact that these drug induced reactions are driven by type IV delayed hypersensitivity reactions. ${ }^{5}$ Unfortunately, in vitro and in vivo studies, in particular, drug provocation tests and allergy testing, have failed to be consistent in identifying the aetiological agent in such cases and hence the diagnosis is made on the basis of exclusion of other causes of rash and on clinical and diagnostic criteria. ${ }^{4,5}$ Nevertheless, improved clinical outcomes after discontinuation of the drug can be helpful in guiding the clinician in identifying the potential offending agent. Based on the clinical presentation, previous drug history and the laboratory data, we concluded that SDRIFE occurred due to the use of valsartan/hydrochlorothiazide use. This diagnosis was strongly supported by the clinical data, the comprehensive histopathological studies and the favourable clinical response after the patient discontinued the antihypertensive therapy.

\section{References}

1. Andersen K, Hjorth N, Menne T. The Baboon syndrome: Systemically induced allergic contact dermatitis. Contact Dermatitis 1984;10:97-100.

2. Häusermann P, Harr T, Bircher AJ. Baboon syndrome resulting from systemic drugs: Is there strife between SDRIFE and allergic contact dermatitis syndrome? Contact Dermatitis 2004;51:297-310.

3. Mofarrah R, Mofarrah R, Kränke B, et al. First report of tamoxifen-induced baboon syndrome. J Cosmet Dermatol 2021;20:2574-8.

4. Roopa B, Kumar SK, Rohini MP, Prasanna V. Case report-baboon syndrome with paracetamol. Int J Basic Clin Pharmacol 2018;7:1-4

5. Elmariah SB, Cheung W, Wang N, et al. Systemic drug-related intertriginous and flexural exanthema (SDRIFE). Dermatol Online J 2009;15:3. 